\title{
Características físicas e químicas de cirigueleiras cultivadas na Zona da Mata Norte de Pernambuco ${ }^{1}$
}

\author{
Quésia Jemima da Silva², Flávia Jamille de Figueiredo³, Vera Lúcia Arroxelas Galvão de Lima*2
}

10.1590/0034-737X201663030002

\section{RESUMO}

A ciriguela (Spondias purpurea L.) é um fruto muito apreciado no nordeste brasileiro, o que tem proporcionado crescente interesse para seu cultivo comercial. Assim, esta pesquisa teve como objetivo avaliar as características físicas e químicas de frutos de 11 genótipos de cirigueleiras, cultivados no Banco Ativo de Germoplasma do Instituto Agronômico de Pernambuco (IPA), localizado na zona da mata Norte de Pernambuco. Frutos maduros foram avaliados quanto à massa, aos diâmetros longitudinal e transversal, ao pH, aos teores de sólidos solúveis (SS), à acidez titulável (AT), ao ácido ascórbico, aos carboidratos solúveis totais e aos carotenoides totais. Com relação às características físicas, os resultados da massa e dos diâmetros longitudinal e transversal não apresentaram diferença estatisticamente significativa. Quanto às características físico-químicas, os resultados demonstraram que o genótipo IPA-1 exibiu o maior teor de ácido ascórbico (32,88 mg 100 g-1 de polpa) e, o genótipo IPA-7, a maior concentração de carotenoides totais, expressa em $\beta$-caroteno (22,63 $\mu \mathrm{g} \mathrm{g}^{-1} \mathrm{de}$ polpa). Os maiores teores de SS e de AT foram apresentados pelos genótipos IPA-9 e IPA-10, respectivamente. O genótipo IPA-4 apresentou maior relação SS/AT, enquanto o genótipo IPA-6 destacou-se dos demais por maior teor de carboidratos solúveis totais, expressos em glicose (32,78\%). Desta forma, pode-se concluir que a variabilidade genética influenciou as características físico-químicas avaliadas.

Palavras-Chave: Spondias purpurea L., ciriguela, qualidade do fruto.

\section{ABSTRACT \\ Physical and chemical characteristics of red mombin trees cultivated in the forest zone in the North of Pernambuco, Brazil}

The red mombin fruit (Spondias purpurea L.) is a very popular fruit in the Brazilian Northeast, which has brought increasing interest for its commercial cultivation. Therefore, the aim of this research was to evaluate the physical and chemical characteristics of red mombin fruits from 11 genotypes cultivated in the Active Germplasm Bank of the Agronomic Institute of Pernambuco (IPA) located in the forest zone in the north of Pernambuco. Mature fruits were evaluated according to their weight, longitudinal and transverse diameter, $\mathrm{pH}$, soluble solids (SS), titratable acidity (TA), ascorbic acid, total soluble carbohydrate and total carotenoids. With respect to the physical characteristics, the results of weight, longitudinal and transverse diameters did not show statistically significant difference. Concerning the chemical characteristics, the results showed that the IPA-1 genotype exhibited the highest ascorbic acid content

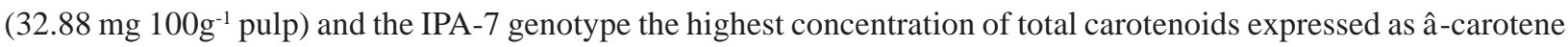

\footnotetext{
Submetido em 15/04/2014 e aprovado em 11/01/2016.

Parte da Dissertação de Mestrado da primeira autora. Pesquisa financiada pelo CNPq

${ }^{2}$ Universidade Federal Rural de Pernambuco, Departamento de Ciências Domésticas, Programa de Pós-Graduação em Ciência e Tecnologia de Alimento, Recife, Pernambuco, Brasil. qjemima@ hotmail.com; veraarroxela@ hotmail.com

${ }_{3}^{3}$ Universidade Federal de Sergipe, Lagarto, Sergipe, Brasil. fjamille@ig.com.br

*Autor para correspondência: veraarroxela@hotmail.com
} 
(22.63 ì $\mathrm{g} \mathrm{g}^{-1}$ pulp). The highest levels of SS and TA were found in the IPA-9 and IPA-10 genotypes, respectively. The IPA-4 genotype showed the highest SS/TA ratio, while the IPA-6 genotype differed from others because of its highest total soluble carbohydrates content expressed as glucose (32.78\%). Therefore, it can be concluded that the genetic variability influenced the physical-chemical characteristics evaluated.

Key words: Spondias purpurea L.; red mombin; fruit quality.

\section{INTRODUÇÃO}

A fruticultura brasileira é um setor da economia que vem registrando significativos avanços, consolidados no aumento da produção e da produtividade e na melhoria da qualidade dos frutos, a exemplo da laranja, da banana, da manga, da uva e da maçã. No entanto, a participação de frutos tropicais, nativos e exóticos nesse mercado é praticamente nula (Lederman et al., 2008).

Dentre as frutas tropicais, destaca-se a ciriguela (Spondias purpurea L.), pertencente à família Anacardiaceae. Considerada uma das frutas mais populares e pequenas da América tropical, a ciriguela adquiriu muitos outros nomes coloquiais, sendo conhecida como ameixa espanhola, ameixa da Espanha, cajá vermelho, ajuela, ciroela, jocote, ciruela mexicana, entre outros (Morton, 1987). No Brasil, este fruto ainda pode ser designado de diferentes formas ortográficas, como: ciriguela (Silva $e t$ al., 2012), siriguela (Todisco, 2012), ciriguela (Lima \& Meleiro, 2012) ou ceriguela (Furtado et al., 2010).

A planta adulta raramente excede a 7,0 metros e seus frutos, isolados ou em cachos, podem, quando maduros, apresentar coloração amarela ou amarelo-avermelhada, medindo de 2,5 a $5 \mathrm{~cm}$ de comprimento e cerca de $2 \mathrm{~cm}$ de diâmetro (Morton, 1987).

Graças à atrativa coloração e ao excelente sabor, esse fruto tem sido comercializado na forma in natura, em diversas regiões do Brasil, notadamente no norte e no nordeste (Martins \& Melo, 2012). No entanto, por ser bastante perecível, pesquisas têm sido conduzidas, visando a seu aproveitamento tecnológico na obtenção de produtos como bebidas fermentadas (Muniz et al., 2002), néctares (Souza Filho et al., 2002), geleia, doce de corte (Lima \& Meleiro, 2012) e polpa em pó (Todisco, 2012).

Estudos sobre as características físico-químicas desse fruto, cultivado na Paraíba, Ceará e Maceió, foram realizados por Filgueiras et al. (2001), Martins et al. (2003) e Omena et al. (2008), respectivamente. No entanto, vários fatores, como condições climáticas, solo, localização, fertilização, estação do ano, cultivares analisados, entre outros, afetam essas características (Harris, 1977).

Tendo em vista que a ciriguela é um fruto típico de regiões semiáridas do Agreste e do Sertão (Lederman et al., 2008), esta pesquisa teve como objetivo avaliar as ca- racterísticas físicas e físico-químicas dos frutos de 11 genótipos de cirigueleiras, cultivados sob as condições edafoclimáticas da Zona da Mata Norte do Estado de Pernambuco.

\section{MATERIAL E MÉTODOS}

Os frutos foram provenientes de 11 genótipos, denominados IPA-1, IPA-2, IPA-3, IPA-4, IPA-5, IPA-6, IPA-7, IPA-8, IPA-9, IPA-10 e IPA-11, pertencentes ao Banco Ativo de Germoplasma de cirigueleira, situado no Campo Experimental do Instituto Agronômico de Pernambuco, localizado no município de Itambé, Pernambuco, sob as coordenadas $7^{\circ} 25^{\prime} \mathrm{Se} 35^{\circ} 06^{\prime} \mathrm{O}$, a $190 \mathrm{~m}$ de altitude (Cavalcante et al., 2013).

Os frutos foram colhidos manualmente nas primeiras horas do dia, no estádio de maturação maduro, durante a safra de verão de 2010. Em seguida, foram acondicionados em sacos plásticos e armazenados sob refrigeração, sendo posteriormente transportados para o Laboratório de Análise Físico-Química de Alimentos, do Departamento de Ciências Domésticas, da Universidade Federal Rural de Pernambuco.

Para a realização das análises físicas, foram utilizados 25 frutos de cada genótipo. A determinação da massa fresca foi efetuada por meio de balança analítica e os diâmetros longitudinal e transversal foram obtidos com paquímetro analógico (Vernier Caliper). Os resultados das variáveis acima foram expressos como média aritmética dos valores obtidos de cada fruto proveniente de cada genótipo correspondente.

Para realização das análises físico-químicas, foi utilizado cerca de $1 \mathrm{~kg}$ do fruto. Após a remoção das sementes com faca de aço inoxidável, as cascas, juntamente com a polpa, foram homogeneizadas em liquidificador doméstico. Após este processo, a polpa obtida (polpa e casca) foi acondicionada em potes plásticos, com tampa de fecho hermético, com capacidade de cerca de $30 \mathrm{~g}$ cada, e armazenada em freezer doméstico $\left(-18^{\circ} \mathrm{C}\right)$ até o momento das análises das características físico-químicas, efetuadas no prazo máximo de um mês.

$\mathrm{O} \mathrm{pH}$ foi determinado diretamente na polpa homogeneizada, utilizando-se potenciômetro (Tecnal, modelo Tec3MP2), previamente calibrado com tampões de $\mathrm{pH} 7$ e 4 , 
segundo técnica do Instituto Adolfo Lutz (1985). O teor de sólidos solúveis (SS) foi obtido por leitura direta em refratômetro portátil (marca ATAGO, N1), com escala variando de 0 a $32^{\circ}$ Brix (AOAC International, 2006). A acidez titulável (AT) foi determinada em $1 \mathrm{~g}$ de polpa diluída em 50 $\mathrm{mL}$ de água destilada, titulando-se com solução de $\mathrm{NaOH}$ 0,1 mol. $\mathrm{L}^{-1}$ até $\mathrm{pH} 8,1$ e os resultados foram expressos em percentagem de ácido cítrico (AOAC International, 2006). A relação SS/AT foi calculada pela razão dos valores obtidos de sólidos solúveis e da acidez titulável.

O teor de ácido ascórbico foi determinado pelo método titulométrico, utilizando-se 2,6 diclorofenol indofenol (AOAC International, 2006) e os resultados foram expressos em mg100 $\mathrm{g}^{-1}$ de polpa.

A extração dos carotenoides foi realizada segundo a metodologia descrita por Rodriguez-Amaya (1999), com leitura de extrato em éter de petróleo e a absorbância registrada no comprimento de onda de $450 \mathrm{~nm}$, em espectrofotômetro Shimadzu UV-1650PC. Durante esta determinação, todas as etapas foram realizadas ao abrigo da luz e o teor de carotenoides totais foi calculado, utilizando-se o coeficiente de absorção de 2500 (Gross, 1987), sendo os resultados expressos em ìg de carotenoides totais em equivalente de $\beta$-caroteno por grama da amostra.

Os carboidratos solúveis totais foram quantificados, utilizando-se o reagente de antrona, o qual se baseia na ação hidrolítica e desidratante do ácido sulfúrico sobre os carboidratos, conforme descrito por Bezerra Neto \& Barreto (2011). A absorbância foi registrada no comprimento de onda de $620 \mathrm{~nm}$, em espectrofotômetro Shimadzu UV1650PC, e os carboidratos solúveis foram quantificados, utilizando-se uma curva-padrão de glicose, sendo os resultados expressos em \% de glicose.

Todas as determinações foram realizadas em triplicata e as médias dos valores encontrados foram submetidas à Análise de Variância (ANOVA) e ao Teste de Tukey, a 5\% de probabilidade, utilizando-se o programa estatístico "Statistica” (versão 5.5, StatSoft, Inc., Tulsa, USA).

\section{RESULTADOS E DISCUSSÃO}

Em relação às análises físicas, os diâmetros longitudinal e transversal e as médias da massa dos frutos dos diferentes genótipos não apresentaram diferenças significativas entre os valores encontrados. Houve apenas diferença $(\mathrm{p}<0,05)$ nos valores da razão entre os diâmetros longitudinal e transversal (Tabela 1).

Embora não tenham apresentado diferenças significativas, os valores de massa dos frutos variaram de $6,5 \mathrm{~g}$ a $8,6 \mathrm{~g}$, sendo o menor e o maior valor encontrados nos frutos dos genótipos IPA-11 e IPA-4, respectivamente. Este parâmetro é uma característica importante para o mercado de frutas frescas, uma vez que, comumente, os frutos mais pesados e maiores tornam-se mais atrativos para os consumidores. Martins et al. (2003), ao avaliarem o desenvolvimento de ciriguelas produzidas no município de Bananeiras, PB, e Filgueiras et al. (2001), em estudo com frutos produzidos no município do Crato, $\mathrm{CE}$, relataram valores médios de massa de 13,98 g e 10,27 g, respectivamente, os quais foram superiores aos encontrados neste trabalho. Valores ainda mais elevados foram encontrados por Hernández et al. (2008), que, ao analisarem os frutos de cirigueleiras cultivadas em diferentes agroecossistemas, encontraram variação de $6,3 \mathrm{~g}$ a $35,8 \mathrm{~g}$.

Os valores dos diâmetros longitudinal (DL) e transversal (DT) dos frutos, neste estudo, variaram de 25,00 mm a 28,00 mm, nos DL, e de $18 \mathrm{~mm}$ a $20 \mathrm{~mm}$, nos DT (Tabela 1). De acordo com Chitarra \& Chitarra (2005), a relação entre os diâmetros longitudinal e transversal determina a forma do fruto, se o diâmetro longitudinal for menor que o diâmetro transversal, o fruto apresentará forma globosa. Neste estudo, a relação DL/DT dos frutos variou de 1,34 a 1,52, indicando forma oblonga. No entanto, Hernández et al. (2008), caracterizando os frutos de cirigueleiras cultivadas em diferentes agroecossistemas, no centro oeste do México, relataram que a relação DL/DT variou de 0,74 a 0,91, o que indica que esses frutos apresentavam a forma globosa.

As características físico-químicas de frutos de genótipos de cirigueleira encontram-se na Tabela 2. Podese observar que não houve diferenças significativas entre os valores de $\mathrm{pH}(3,3$ a 3,4$)$. Valores próximos foram reportados por Filgueiras et al. (2001), que, ao estudarem a qualidade de ciriguelas, relataram valores de $\mathrm{pH}$ de 3,44 , em ciriguelas no estádio de maturação maduro. No estudo de Hernández et al. (2008) foram encontrados valores de $\mathrm{pH}$ entre 2,7 e 3,5 .

Os teores de sólidos solúveis (SS), expressos em ${ }^{\circ}$ Brix, apresentaram diferença significativa (Tabela 2), e seus valores variaram de 15,8 (IPA-11) a 17,7 (IPA-9). Valor mais elevado foi encontrado em ciriguelas oriundas de Crato, $\mathrm{CE}$, que, no estádio de maturação maduro, mostraram teor de sólidos solúveis de 21,25 ${ }^{\circ} \mathrm{Brix}$ (Filgueiras et al., 2001). Freire et al. (2011), avaliando frutos de ciriguela em seis estádios de maturação, coletados de diferentes plantas no município de Pombal, PB, também encontraram valor mais elevado, de sólidos solúveis, sendo, em frutos maduros, de $21,00 \%$.

Com relação à acidez titulável, constata-se na Tabela 2 que houve diferença significativa entre os teores, com destaque para o genótipo IPA-10 (1,08\%) que apresentou o mais alto percentual. No entanto, Sacramento et al. (2008), em estudos efetuados com ciriguelas produzidas na Bahia, revelaram valor de acidez titulável de $0,67 \%$, bem mais baixo do que os encontrados neste estudo.

A relação SS/AT, calculada neste estudo, mostrou diferença estatística entre os resultados encontrados (Ta- 
bela 2), com destaque para o genótipo IPA-4, que apresentou o mais alto valor (18,21). Freire et al. (2011), que, avaliando frutos de ciriguela, em seis estádios de maturação, e coletados de diferentes plantas, no município de Pombal, PB, relataram que em frutos maduros o valor dessa relação foi de 35,62\%. A relação SS/AT é um parâmetro de qualidade para aceitação de frutas, sendo um importante indicativo do sabor, pois relaciona os açúcares e os ácidos do fruto. Portanto, considera-se que quanto mais elevados os valores da relação SS/AT, mais doces são os frutos.

Na Tabela 3, encontram-se os teores de ácido ascórbico,

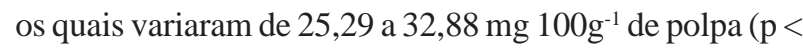
0,05), quantificados nos frutos dos genótipos IPA-3 e IPA1, respectivamente. Essa vitamina ocorre naturalmente nas frutas e hortaliças, sendo um parâmetro nutricional de grande importância, por causa de seu elevado poder antioxi- dante, na prevenção e combate de diversas doenças, especialmente o escorbuto (Galisa et al., 2008). Filgueiras et al. (2001) relataram que nos frutos de cirigueleira em estádio de maturação maduro, teor de ácido ascórbico encon-

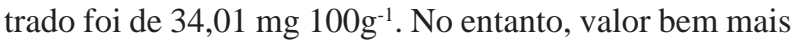
elevado de ácido ascórbico foi relatado por López et al. (2004) em ciriguelas maduras cultivadas no México (67,3 $\left.\mathrm{mg} 100 \mathrm{~g}^{-1}\right)$.

Quanto aos teores de carboidratos solúveis (Tabela 3), expressos em \% de glicose, pode-se observar que houve diferenças estatísticas entre os genótipos estudados. Os teores encontrados variaram de 17,88\%, nos frutos do genótipo IPA-7, a 32,78\%, nos frutos do genótipo IPA-6, sendo esse valor estatisticamente diferente dos demais. Filgueiras et al. (2001) relataram, em frutos maduros, teor de $18,68 \%$ de açúcares solúveis, sendo esse valor próximo aos encontrados neste estudo.

Tabela 1: Características físicas dos frutos de genótipos de cirigueleira provenientes do Banco Ativo de Germoplasma do IPA (Itambé, PE, safra 2010)

\begin{tabular}{lcccc}
\hline Genótipos & MF $(\mathbf{g})$ & DL $(\mathbf{m m})$ & DT $(\mathbf{m m})$ & DL/DT \\
\hline IPA-1 & $7,4 \pm 1,67 \mathrm{a}$ & $26,0 \pm 0,26 \mathrm{a}$ & $19,0 \pm 0,17 \mathrm{a}$ & $1,34 \pm 0,09 \mathrm{bc}$ \\
IPA-2 & $7,7 \pm 1,68 \mathrm{a}$ & $27,0 \pm 0,35 \mathrm{a}$ & $19,0 \pm 0,18 \mathrm{a}$ & $1,44 \pm 0,12 \mathrm{abc}$ \\
IPA-3 & $7,1 \pm 1,70 \mathrm{a}$ & $27,0 \pm 0,23 \mathrm{a}$ & $19,0 \pm 0,19 \mathrm{a}$ & $1,42 \pm 0,11 \mathrm{abc}$ \\
IPA-4 & $8,6 \pm 1,70 \mathrm{a}$ & $28,0 \pm 0,22 \mathrm{a}$ & $20,0 \pm 0,13 \mathrm{a}$ & $1,38 \pm 0,07 \mathrm{bc}$ \\
IPA-5 & $8,2 \pm 1,37 \mathrm{a}$ & $28,0 \pm 0,19 \mathrm{a}$ & $20,0 \pm 0,11 \mathrm{a}$ & $1,40 \pm 0,07 \mathrm{abc}$ \\
IPA-6 & $8,0 \pm 1,45 \mathrm{a}$ & $27,0 \pm 0,29 \mathrm{a}$ & $19,0 \pm 0,14 \mathrm{a}$ & $1,44 \pm 0,14 \mathrm{abc}$ \\
IPA-7 & $8,4 \pm 1,90 \mathrm{a}$ & $27,0 \pm 0,34 \mathrm{a}$ & $20,0 \pm 0,31 \mathrm{a}$ & $1,36 \pm 0,13 \mathrm{c}$ \\
IPA-8 & $8,5 \pm 2,12 \mathrm{a}$ & $28,0 \pm 0,31 \mathrm{a}$ & $19,0 \pm 0,24 \mathrm{a}$ & $1,44 \pm 0,10 \mathrm{abc}$ \\
IPA-9 & $7,1 \pm 1,39 \mathrm{a}$ & $27,0 \pm 0,17 \mathrm{a}$ & $18,0 \pm 0,14 \mathrm{a}$ & $1,52 \pm 0,10 \mathrm{a}$ \\
IPA-10 & $6,7 \pm 1,46 \mathrm{a}$ & $27,0 \pm 0,19 \mathrm{a}$ & $18,0 \pm 0,14 \mathrm{a}$ & $1,50 \pm 0,13 \mathrm{ab}$ \\
IPA-11 & $6,5 \pm 1,56 \mathrm{a}$ & $25,0 \pm 0,24 \mathrm{a}$ & $18,0 \pm 0,22 \mathrm{a}$ & $1,40 \pm 0,10 \mathrm{abc}$ \\
\hline
\end{tabular}

Valores médios \pm desvio padrão. Médias nas colunas seguidas por letras iguais não apresentam diferença significativa pelo Teste de Tukey a $5 \%$ de probabilidade.

MF = Massa do Fruto; DL = Diâmetro Longitudinal; DT = Diâmetro Transversal; DL/DT = Razão Diâmetro Longitudinal e Diâmetro transversal.

Tabela 2: Características físico-químicas de frutos de genótipos de cirigueleira provenientes do Banco Ativo de Germoplasma do IPA (Itambé, PE, safra 2010)

\begin{tabular}{|c|c|c|c|c|}
\hline Genótipos & pH & SS ( ${ }^{\circ}$ Brix) & AT (\% ác. cítrico) & SS/AT \\
\hline IPA-1 & $3,3 \pm 0,01 \mathrm{a}$ & $16,5 \pm 0,46 \mathrm{bc}$ & $0,94 \pm 0,49 \mathrm{c}$ & $17,55 \pm 0,43 \mathrm{abc}$ \\
\hline IPA-2 & $3,4 \pm 0,04 \mathrm{a}$ & $17,2 \pm 0,29 a b$ & $0,99 \pm 0,49 \mathrm{c}$ & $17,37 \pm 0,26 a b c$ \\
\hline IPA-3 & $3,3 \pm 0,03 \mathrm{a}$ & $17,0 \pm 0,20 \mathrm{ab}$ & $1,05 \pm 0,59 \mathrm{ab}$ & $16,19 \pm 0,53 \mathrm{~cd}$ \\
\hline IPA-4 & $3,4 \pm 0,05 \mathrm{a}$ & $17,3 \pm 0,31 a b$ & $0,95 \pm 0,49 \mathrm{c}$ & $18,21 \pm 0,12 \mathrm{a}$ \\
\hline IPA-5 & $3,3 \pm 0,01 \mathrm{a}$ & $15,9 \pm 0,12 b c$ & $0,95 \pm 0,49 \mathrm{c}$ & $16,74 \pm 0,26 \mathrm{bcd}$ \\
\hline IPA-6 & $3,3 \pm 0,03 \mathrm{a}$ & $16,4 \pm 0,40 \mathrm{bc}$ & $1,00 \pm 0,49 b c$ & $16,40 \pm 1,12$ bcd \\
\hline IPA-7 & $3,3 \pm 0,01 \mathrm{a}$ & $16,9 \pm 0,12 b$ & $0,94 \pm 0,49 \mathrm{c}$ & $17,98 \pm 0,40 a b$ \\
\hline IPA-8 & $3,3 \pm 0,01 \mathrm{a}$ & $16,9 \pm 0,12 \mathrm{ab}$ & $0,94 \pm 0,49 \mathrm{c}$ & $17,98 \pm 0,50 \mathrm{ab}$ \\
\hline IPA-9 & $3,3 \pm 0,01 \mathrm{a}$ & $17,7 \pm 0,12 \mathrm{a}$ & $1,05 \pm 0,59 a b$ & $16,85 \pm 0,18 a b c$ \\
\hline IPA-10 & $3,3 \pm 0,01 \mathrm{a}$ & $17,4 \pm 0,40 a$ & $1,08 \pm 0,59 \mathrm{a}$ & $16,11 \pm 0,70 \mathrm{~cd}$ \\
\hline IPA-11 & $3,3 \pm 0,01 \mathrm{a}$ & $15,8 \pm 0,20 \mathrm{c}$ & $1,03 \pm 0,59 \mathrm{ab}$ & $15,34 \pm 0,38 \mathrm{~d}$ \\
\hline
\end{tabular}

Valores médios \pm desvio padrão

Médias nas colunas seguidas por letras iguais não apresentam diferença significativa pelo Teste de Tukey a 5\% de probabilidade. SS = Sólidos Solúveis; AT = Acidez Titulável; SS/AT = Índice de Sabor.

Rev. Ceres, Viçosa, v. 63, n.3, p. 285-290, mai/jun, 2016 
Tabela 3: Teores de ácido ascórbico, carboidratos solúveis e carotenoides totais de frutos de genótipos de cirigueleira provenientes do Banco Ativo de Germoplasma do IPA, Itambé, PE, safra verão de 2010

\begin{tabular}{lccc}
\hline Genótipos & $\begin{array}{c}\text { Ácido Ascórbico } \\
\left(\mathbf{m g ~ 1 0 0 g}^{-1}\right)\end{array}$ & $\begin{array}{c}\text { Carboidratos Solúveis } \\
(\boldsymbol{\%} \mathbf{d e} \text { glicose })\end{array}$ & $\begin{array}{c}\text { Carotenoides Totais } \\
\left(\boldsymbol{\mu g} \text { de } \boldsymbol{\beta} \text {-caroteno } \mathbf{g}^{-1} \mathbf{d e} \text { polpa }\right)\end{array}$ \\
\hline IPA-1 & $32,88 \pm 2,5 \mathrm{a}$ & $26,80 \pm 0,7 \mathrm{~b}$ & $15,11 \pm 0,4 \mathrm{~cd}$ \\
IPA-2 & $29,89 \pm 0,5 \mathrm{a}$ & $20,03 \pm 3,0 \mathrm{c}$ & $16,64 \pm 0,8 \mathrm{~b} \mathrm{c}$ \\
IPA-3 & $25,29 \pm 0,9 \mathrm{c}$ & $24,25 \pm 1,9 \mathrm{bc}$ & $18,21 \pm 1,2 \mathrm{~b}$ \\
IPA-4 & $25,48 \pm 0,9 \mathrm{bc}$ & $22,61 \pm 0,3 \mathrm{bc}$ & $18,40 \pm 0,4 \mathrm{~b}$ \\
IPA-5 & $26,19 \pm 0,3 \mathrm{~b}$ & $18,43 \pm 1,2 \mathrm{de}$ & $10,03 \pm 1,2 \mathrm{~d}$ \\
IPA-6 & $25,84 \pm 0,7 \mathrm{~b}$ & $32,78 \pm 1,9 \mathrm{a}$ & $20,91 \pm 0,7 \mathrm{a}$ \\
IPA-7 & $25,71 \pm 0,5 \mathrm{~b}$ & $17,88 \pm 0,9 \mathrm{e}$ & $22,63 \pm 2,0 \mathrm{a}$ \\
IPA-8 & $26,11 \pm 0,5 \mathrm{~b}$ & $21,27 \pm 0,4 \mathrm{c}$ & $16,40 \pm 2,3 \mathrm{c}$ \\
IPA-9 & $26,45 \pm 2,4 \mathrm{ab}$ & $19,39 \pm 0,5 \mathrm{~d}$ & $16,85 \pm 0,05 \mathrm{bc}$ \\
IPA-10 & $25,96 \pm 0,2 \mathrm{~b}$ & $21,98 \pm 0,6 \mathrm{bc}$ & $13,40 \pm 0,04 \mathrm{~d}$ \\
IPA-11 & $25,76 \pm 0,9 \mathrm{~b}$ & $23,47 \pm 4,2 \mathrm{~b}$ & $14,15 \pm 0,8 \mathrm{~d}$ \\
\hline
\end{tabular}

Valores médios \pm desvio padrão

Médias nas colunas seguidas por letras iguais não apresentam diferença significativa pelo Teste de Tukey a 5\% de probabilidade.

Os carotenoides são importantes não apenas como precursores de vitamina A, mas também porque apresentam considerável atividade antioxidante (Rufino et al., 2010). Foi constatada diferença significativa nos teores de carotenoides dos frutos, expressos em $\mu \mathrm{g}$ de $\beta$-caroteno $\mathrm{g}^{-1}$ de polpa, em função do genótipo (Tabela 3). O genótipo IPA-5 apresentou o mais baixo teor de carotenoides totais (10,03 $\left.\mu \mathrm{g} \mathrm{g}^{-1}\right)$, enquanto o genótipo IPA-7 apresentou o teor mais elevado (22,63 $\left.\mu \mathrm{g} \mathrm{g}^{-1}\right)$. Segundo Rodriguez-Amaya et al. (2008), apenas alimentos que contenham mais de 20 $\mu \mathrm{g} \mathrm{g}^{-1}$ de carotenoides são importantes para a saúde. Assim, o consumo de ciriguela pode contribuir para o aporte nutricional de carotenoides.

Os valores de carotenoides totais encontrados neste estudo mostram-se próximos ao relatado por Murillo et al. (2010), que, em ciriguelas do Panamá, detectaram um teor de $18,20 \mu \mathrm{g} \mathrm{g}^{-1}$. No entanto, Neves et al. (2008) encontraram, em ciriguelas maduras comercializadas na feira livre da Cidade de Recife, valor mais elevado (28,5 $\left.\mu \mathrm{g} \cdot \mathrm{g}^{-1}\right)$.

O conteúdo de carotenoides dos vegetais de uma mesma espécie pode ser afetado por vários fatores, como grau de maturação, condições climáticas, tipo de solo e condições de cultivo, exposição à luz solar, processamento e estocagem, entre outros (Rodriguez-Amaya, 1999).

\section{CONCLUSÕES}

Os frutos dos genótipos de ciriguelas avaliados não apresentam diferenças significativas nos parâmetros físicos de massa, diâmetro longitudinal e transversal; a relação entre os diâmetros longitudinal e transversal mostrou que esses frutos apresentam a forma oblonga.

Em relação aos parâmetros químicos, o genótipo IPA-9 apresentou o maior teor de sólidos solúveis, o IPA-4 a maior relação SS/AT, o IPA-6 o maior teor de carboidratos solúveis totais, o IPA-1 o maior teor de ácido ascórbico e o
IPA-7 a maior concentração de carotenoides totais, mostrando que a variabilidade genética influenciou as características químicas avaliadas.

Considerando-se as características nutricionais avaliadas neste estudo, é possível indicar como o melhor genótipo de ciriguela o IPA-7, tendo em vista que seus frutos exibiram a maior concentração de carotenoides totais e expressivo teor de ácido ascórbico.

\section{AGRADECIMENTOS}

As autoras agradecem à FACEPE (Fundação de Amparo à Ciência e Tecnologia do Estado de Pernambuco), pela concessão de bolsa de mestrado, e ao CNPq (Conselho Nacional de Desenvolvimento Científico e Tecnológico), pelo apoio financeiro ao projeto de pesquisa sobre frutos do gênero Spondias cultivados na zona da Mata de Pernambuco (Projeto Casadinho, Edital 16/2008, Processo $\left.n^{\circ} 620007 / 2008\right)$.

\section{REFERÊNCIAS}

AOAC - Association of Official Analytical Chemists (2006) Official Methods of Analysis of Association of Official Analytical Chemists International. $18^{\text {th }}$ ed. Gaithersburg, AOAC International. $158 \mathrm{p}$.

Bezerra Neto E \& Barreto LP (2011) Análises químicas e bioquímicas em plantas. Recife, UFRPE. 267p.

Cavalcante M, Lira MA, Santos MVF, Santoro KR, Ferreira RLC \& Leão Neto JMC (2013) Potencial produtivo de Pennisetum spp. sob níveis de nitrogênio na Zona da Mata de Pernambuco. Archivos de Zootecnia, 62:501-512.

Chitarra MIF \& Chitarra AB (2005) Pós-colheita de frutos e hortaliças: fisiologia e manuseio. $2^{\mathrm{a}}$ ed. Lavras, UFLa. 785p.

Filgueiras HAC, Alves RE, Moura CFH, Nágela ACO \& Araújo NCC (2001) Calidad de frutas nativas de latinoamerica para indústria: ciruela mexicana (Spondias purpurea L.). Proceedings of the Interamerican Society for Tropical Horticulture, 43:68-71.

Rev. Ceres, Viçosa, v. 63, n.3, p. 285-290, mai/jun, 2016 
Freire ECBS, Silva FVG, Santos AF \& Medeiros IF (2011) Avaliação da qualidade de ciriguela (Spondias purpurea L.) em diferentes estádios de maturação. Revista Verde, 6:27-40.

Furtado GF, Silva FS, Porto AG \& Santos P (2010) Secagem de polpa de ceriguela pelo método de camada de espuma. Revista Brasileira de Produtos Agroindustriais, 12:9-14.

Galisa MS, Esperança LMB \& Sá NG (2008) Nutrição-conceitos e aplicações. São Paulo, M. Books do Brasil Editora Ltda. 258p.

Gross J (1987) Carotenoids. In: Gross J (Ed.) Pigments in fruits. London, Academic Press. p.87-186.

Harris RS (1977) Effects of agricultural practices on foods of plant origin. In: Harris RS \& Karmas E (Eds.) Nutritional evaluation of food processing. Connecticut, The Avi Publishing Company. p.33-57.

Hernández BCR, Eulogio PB, Ramos JZC, Urias AM, Hasbach GP \& Barrios EP (2008) Sistemas de producción de Spondias purpurea (Anacardiaceae) en el centro-occidente de México. Revista de Biología Tropical, 56:675-687.

Instituto Adolfo Lutz (1985) Normas Analíticas do Instituto Adolfo Lutz: métodos químicos e físicos para análise de alimentos. $3^{a}$ ed. São Paulo, Instituto Adolfo Lutz. 533p.

Lederman IE, Silva Júnior JF, Bezerra JEF \& Lira Júnior JS (2008) Potencialidade das espécies de Spondias no desenvolvimento da fruticultura brasileira. In: Lederman IE, Lira Júnior JS \& Silva Júnior JF (Eds.) Spondias no Brasil: Umbú, Cajá e Espécies Afins. Recife, Editora Universitária da UFRPE. p.15-22.

Lima ICGS \& Meleiro CHA (2012) Desenvolvimento, avaliação físico-química e sensorial de geleia e doce de corte de ciriguela (Spondias purpurea L.) visando o crescimento da cadeia produtiva do fruto. Boletim do Centro de Pesquisa de Processamento de Alimentos, 30:221-232.

López AP, Veloz CS, Galarza MLA \& Lúa AM (2004) Efecto del grado de madurez en la calidad y vida postcosecha de ciruela mexicana (Spondias purpurea L.). Revista Fitotecnia Mexicana, 27:133-139.

Martins LP, Silva SM, Alves RE \& Filgueiras HAC (2003) Desenvolvimento de frutos de cirigueleira (Spondias purpurea L.). Revista Brasileira de Fruticultura, 25:11-14

Martins ST \& Melo B (2012) Spondias (Cajá e outras). Disponível em: <http://www.fruticultura.iciag.ufu.br/caja.html>. Acessado em: 27 de junho de 2012.

Morton JF (1987) Purple mombin (Spondias purpurea L.) Disponível em <http://www.hort.purdue.edu/newcrop/morton/ purple_mombin.html>. Acessado em: 30 de agosto de 2012.
Muniz CR, Borges MF, Abreu FAP, Nassu RT \& Freitas CAS (2002) Bebidas fermentadas a partir de frutos tropicais. Boletim do Centro de Pesquisa de Processamento de Alimentos, 20:309322.

Murillo E, Meléndez-Martínez AJ \& Portugal F (2010) Screening of vegetables and fruits from Panama for rich sources of lutein and zeaxanthin. Food Chemistry, 122:167-172.

Neves MVM, Silva QJ, Melo EA \& Lima VLAG (2008) Caracterização físico-química de ciriguela (Spondias purpurea L.) comercializada na feira livre de Casa Amarela, Recife - PE. In: Simpósio Brasileiro Sobre Umbu, Cajá e Espécies Afins, Recife. Anais, Empresa Pernambucana de Pesquisa Agropecuária-IPA/ UFRPE. CD-ROM.

Omena CMB, Oliveira MBF \& Costa JG (2008) Caracterização de frutos de cirigueleira (Spondias purpurea L.) comercializado em Maceió, Alagoas. In: $20^{\circ}$ Congresso Brasileiro de Fruticultura, Vitória. Anais, Sociedade Brasileira de Fruticultura. CD-ROM.

Rodriguez-Amaya DB (1999) A Guide to Carotenoids Analysis in Food. Washington, ILSI Press. 64p.

Rodriguez-Amaya DB, Kimura M \& Amaya-Farfan J (2008) Fontes brasileiras de carotenoides: tabela brasileira de composição de carotenoides em alimentos. Brasília, MMA/SBF. 99p.

Rufino MSM, Alves RE, Brito ES, Pérez-Jiménez J, Saura-Calixto F \& Mancini-Filho J (2010) Bioactive compounds and antioxidant capacities of 18 non-traditional tropical fruits from Brazil. Food Chemistry, 121:996-1002.

Sacramento CK, Ahnert D, Barretto WS \& Faria JC (2008) Recursos genéticos e melhoramento de Spondias na Bahia- cajazeira, cirigueleira e cajaraneira. In: Lederman IE, Lira Júnior JS \& Silva Júnior JF (Eds.) Spondias no Brasil: Umbú, Cajá e Espécies Afins. Recife, Editora Universitária da UFRPE. p.54-62.

Souza Filho MSM, Lima JR, Nassu RT, Borges MF \& Moura CFH (2002) Avaliação físico-química e sensorial de néctares de frutas nativas da região norte e nordeste do Brasil: estudo exploratório. Brazilian Journal of Food Technology, 5:139143.

Silva QJ, Moreira ACCG, Melo EA \& Lima VLAG (2012) Compostos fenólicos e atividade antioxidante de genótipos de ciriguelas (Spondia Purpurea L.). Alimentos e Nutrição, 23:7380 .

Todisco KM (2012) Polpa de siriguela (Spondias purpurea L.) em pó atomizada: caracterizações físicas, físico-químicas, compostos bioativos e avaliação do comportamento higroscópico. Dissertação de Mestrado. Universidade Estadual de Maringá, Maringá. 70p. 